\title{
Hinweise für die Benutzung des Wörterbuches
}

1. Vor Benutzung des Buches mache man sich vertraut:

a) mit der Schreibweise (S. VII und VIII);

b) mit den Abkürzungen (S. X, XI und XII);

c) mit der Anleitung zum Lesen lateinischer und griechischer Wörter (S. IX und XV).

2. Die Finordnung eines Stichwortes geschieht nach der Buchstabenreihe $(\mathrm{ABC})$, und zwar nach der deutsehen Schreibweise des Wortes, also Appendizitis, Akne, Alopezie. Vgl. den Abschnitt Schreibweise (S. VII u. VIII). Zweiteilige Wörter (Beispiel: H-Antigen) werden wie einteilige behandelt (Hantigen).

3. Findet man ein Wort nicht an der Stelle, die ihm nach der Schreibweise mit $\mathbf{k}$ oder $\mathbf{z}$ zukommt, so ist unter $\mathbf{c}$ nachzusehen und umgekehrt.

4. Die Laute $\ddot{\mathbf{a}}, \ddot{\boldsymbol{o}}$, ï, äu sind hinsichtlich der Reihenfolge so behandelt, wie es der Schreibweise ae, oe, ue, aeu entspricht.

5. Die Buchstaben $\mathbf{i}$ und $\mathbf{j}$ sind getrennt behandelt.

6. Ein * hinter einem Wort bedeutet, daß dieses an der ihm nach alphabetischer Reihenfolge zukommenden Stelle nachzulesen ist. Bei Nerven (Sympathikus) bedeutet es den Ursprung und das Wurzelgebiet.

Ein $\rightarrow$ bei Nerven bedeutet den Verlauf.

7. Ein Apostroph hinter Eigennamen oder eckigen Klammern bedeutet scher, sche oder sches. Und zwar wurde er nur hinter Autorennamen gesetzt; Patientennamen erhielten keinen Apostroph, sondern einen Bindestrich (Beispiel: Duffy-System).

8. Daß ein Wort aus dem Griechischen stammt, ist aus den zur etymologischen Erklärung benutzten Schriftzeichen zu ersehen. Fremdwörter, bei denen hinter der etymologischen Erklärung die Angabe der Sprache fehlt, stammen aus dem Lateinischen. 
Bei den übrigen Wörtern ist die Sprache, aus der sie stammen, abgekürzt angegeben.

9. Fehlt bei einer etymologischen Angabe die deutsche Übersetzung, so ist die folgende Erklärung mit der Übersetzung gleichbedeutend.

10. Bei zusammengesetzten Wörtern sind die Teile des Wortes einzeln nachzulesen.

11. Fehlt bei einem Wort oder dem Bestandteil eines Wortes die Angabe der Etymologie, so ist diese bei den voranstehenden oder den folgenden Wörtern zu finden.

12. Die Vorsilben M, Me, Mac, 0' gelten als untrennbare Bestandteile des Namens, z. B. ist Mac Burney unter M zu suchen.

13. Die hinter einem Eigennamen aufgeführte Stadt ist diejenige, in der der Betreffende sich hauptsächlich oder zuletzt aufgehalten hat.

14. Erläuterungen zu Eigennamen finden sich meist nur an der Stelle, an der der Name alphabetisch auftritt. An anderen Stellen ist gewöhnlich auf eine Erläuterung verzichtet worden. Stehen hinter dem Eigennamen nur Vorname und Berufsbezeichnung, so handelt es sich in der Regel um Zeitgenossen. 


\section{Anleitung zur Betonung lateinischer Wörter}

1. Bei zweisilbigen Wörtern wird die erste Silbe betont.

2. Bei mehrsilbigen Wörtern wird die vorletzte Silbe betont. Ist diese kurz, so wird die drittletzte Silbe betont.

3. Als lang gelten alle Silben mit einem langen Vokal. Ist er kurz und folgen auf ihn mindestens zwei Konsonanten, so gilt die Silbe dennoch als lang.

\section{Die lateinischen Grundzahlen von 1-1000}

\begin{tabular}{|c|c|c|c|c|c|}
\hline 1 & I & unus (una, unum) & 28 & XXVIII & duodetriginta \\
\hline 2 & II & duo (duae, duo) & 29 & XXVIIII & undetriginta \\
\hline 3 & III & tres (tria) & & (XXIX) & \\
\hline 4 & IIII (IV) & quattuor & 30 & $\mathrm{XXX}$ & triginta \\
\hline 5 & V & quinque & 33 & XXXIII & tres et triginta \\
\hline 6 & VI & $\operatorname{sex}$ & & & triginta tres \\
\hline 7 & VII & septem & 40 & XXXX & quadraginta \\
\hline 8 & VIII & octo & & $(\mathrm{XL})$ & \\
\hline 9 & VIIII (IX) & novem & 50 & $\mathrm{~L}$ & quinquaginta \\
\hline 10 & $\mathrm{X}$ & decem & 60 & $\mathrm{LX}$ & sexaginta \\
\hline 11 & XI & underim & 70 & LXX & septuaginta \\
\hline 12 & XII & duodecim & 80 & LXXX & octoginta \\
\hline 13 & XIII & tredecim & 90 & LXXXX & nonaginta \\
\hline 14 & XIIII(XIV) & quattuordecim & & (XC) & \\
\hline 15 & $\mathrm{XV}$ & quindecim & 100 & $\mathrm{C}$ & centum \\
\hline 16 & XVI & sedecim & 101 & CI & centum unus \\
\hline 17 & XVII & septendecim & 200 & $\mathrm{CC}$ & ducenti, ae, a \\
\hline 18 & XVIII & duodeviginti & 300 & $\mathrm{CCC}$ & trecenti, ae, a \\
\hline 19 & XVIIII & undeviginti & 400 & CD & quadringenti,ae, \\
\hline & (XIX) & & 500 & $\mathrm{D}$ & quingenti, ae, a \\
\hline 20 & $\mathrm{XX}$ & viginti & 600 & DC & sescenti, ae, a \\
\hline 21 & XXI & unus et viginti, & 700 & DCC & septingenti, ae, a \\
\hline & & viginti unus & 800 & DCCC & octingenti, ae, a \\
\hline & XXII & $\begin{array}{c}\text { duo et viginti, } \\
\text { viginti duo }\end{array}$ & $\begin{array}{r}900 \\
1000\end{array}$ & $\begin{array}{l}\mathrm{CM} \\
\mathrm{M}\end{array}$ & $\begin{array}{l}\text { nongenti, ae, a } \\
\text { mille }\end{array}$ \\
\hline
\end{tabular}

